\title{
Evaluation of serum omentin-1 and apelin concentrations in patients with hidradenitis suppurativa
}

\author{
Marcos A. González-López ${ }^{1}$, J. Gonzalo Ocejo-Vińals², Cristina Mata³ , Diego Díaz¹, Sandra Guiral², Virginia Portilla4, \\ Alfonso Corrales ${ }^{4}$, M. Carmen González-Vela5, Miguel A. González-Gay4, Ricardo Blanco ${ }^{4}$, José L Hernández ${ }^{6}$
}

${ }^{1}$ Division of Dermatology, Hospital Universitario Marqués de Valdecilla, University of Cantabria, IDIVAL, Santander, Cantabria, Spain ${ }^{2}$ Division of Immunology, Hospital Universitario Marqués de Valdecilla, University of Cantabria, IDIVAL, Santander, Cantabria, Spain ${ }^{3}$ Division of Rheumatology, Hospital Comarcal, Laredo, Cantabria, Spain

${ }^{4}$ Division of Rheumatology, Hospital Universitario Marqués de Valdecilla, University of Cantabria, IDIVAL, Santander, Cantabria, Spain ${ }^{5}$ Division of Pathology, Hospital Universitario Marqués de Valdecilla, University of Cantabria, IDIVAL, Santander, Cantabria, Spain ${ }^{6}$ Division of Internal Medicine, Hospital Universitario Marqués de Valdecilla, University of Cantabria, IDIVAL, Santander, Cantabria, Spain

Adv Dermatol Allergol 2021; XXXVIII (3): 450-454

DOI: https://doi.org/10.5114/ada.2021.107932

\begin{abstract}
Introduction: Recent studies suggest a role of adipokines in the pathogenesis of hidradenitis suppurativa (HS). Omentin- 1 and apelin are two recently identified adipokines that have been involved in the regulation of metabolic and inflammatory responses.

Aim: To investigate serum omentin-1 and apelin levels in patients with HS and to assess their associations with metabolic parameters, disease severity and HS risk.

Material and methods: This case-control study included 139 non-diabetic individuals (78 HS patients and 61 ageand sex-matched controls). Serum concentrations of omentin-1 and apelin and the homeostasis model assessment of insulin resistance (HOMA-IR) were measured in all participants.

Results: Serum omentin-1 concentrations were significantly higher in HS patients compared to controls, whereas apelin serum levels did not significantly differ between both groups. These differences in omentin-1 concentrations remained significant even after adjusting for age, sex, and body mass index (BMI). The results of logistic regression analysis showed that increased omentin-1 plasma levels were an independent risk factor for HS. However, we found no association between serum levels of both omentin-1 and apelin with HS severity.

Conclusions: Our results show that patients with HS have raised omentin-1 serum levels, which are associated with HS risk.
\end{abstract}

Key words: hidradenitis suppurativa, apelin, omentin, adipokines.

\section{Introduction}

Hidradenitis suppurativa/acne inversa (HS) is a chronic, inflammatory, follicular disease, clinically characterized by recurrent, painful, deep-seated inflamed lesions predominantly located in the inverse areas of the body [1]. HS is currently recognized as a systemic inflammatory disease linked to several comorbidities [2, 3]. In this regard, the association between HS and metabolic conditions such as insulin resistance (IR) [4], metabolic syndrome (MS) [5] and type 2 diabetes mellitus (T2DM) [6] has been established. Moreover, patients with HS have an increased cardiovascular (CV) risk [7].
The etiopathogenesis of HS still remains uncertain, although it is considered to be multifactorial. Current evidence suggests the presence of an altered immune response in HS, with enhanced expression of cytokines, such as tumour necrosis factor- $\alpha$ (TNF- $\alpha$ ), interleukin (IL)-1 $\beta$ and IL-17 in lesional skin and in the serum of HS patients [8-10].

Adipokines are biologically active polypeptide hormones, mainly produced by adipocytes and adipose tissue-associated macrophages (ATMs) [11]. There is a growing body of evidence indicating that these pleiotropic molecules play a pivotal role in the regulation of

Address for correspondence: Marcos A. González-López MD, PhD, Servicio de Dermatología, Hospital Universitario Marqués de Valdecilla, Avda, de Valdecilla s/n, E-39008, Santander, Spain, phone: 942202523, e-mail: marcosg@aedv.es Received: 7.01.2020, accepted: 20.01.2020. 
the immune-mediated mechanisms and inflammatory responses. Furthermore, dysregulation of adipokine secretion has been implicated in the pathogenesis of several chronic inflammatory diseases [11]. In this respect, it has been recently reported that adiponectin, leptin, resistin, and visfatin are dysregulated in patients with HS [12-14]. In this sense, it has also been reported that resistin and visfatin might be implicated in HS pathogenesis $[13,14]$.

Omentin-1 and apelin are two recently identified adipokines that have been involved in inflammatory and metabolic processes $[15,16]$. However, to our knowledge, there are no data in the medical literature about the expression level and potential pathogenic role of these adipokines in $\mathrm{HS}$.

\section{Aim}

The aim of this study was to analyse the serum concentrations of omentin-1 and apelin in a series of HS patients and to investigate the possible relationships of these adipokines and metabolic parameters, disease severity and the risk of HS.

\section{Material and methods}

\section{Study participants and protocol}

We carried out a case-control study that included 78 patients with HS and 61 age- and sex-matched controls. HS patients were recruited from our dermatology outpatient clinic at the University Hospital Marques de Valdecilla (Santander, Northern Spain). All of the patients fulfilled the established diagnostic criteria of HS. The control group comprised hospital medical staff and subjects who had been admitted to the dermatology department for non-inflammatory disorders, such as melanocytic nevus, warts or epithelioma. The research protocol was approved by the local institutional ethics committee, and it complied with the ethical standards outlined in the Declaration of Helsinki. Patients gave informed consent to participate in this study.

For the purpose of this study, we used the methodology previously reported in detail [14]. Subjects were excluded from our study if they met any of the following criteria: age $<18$ years, documented history of CV events, type 1 or T2DM and other endocrine diseases that might influence glucose metabolism, chronic kidney or liver diseases, treatment with drugs affecting carbohydrate metabolism in the previous 6 months, and the presence of concomitant inflammatory cutaneous or systemic disorders.

The severity of HS was assessed by the HS Physician Global Assessment (HS-PGA), which includes 6 stages (scale $0 \pm 5$ ) with clear guidance for disease severity assessment, and the Hurley staging. According to HS-PGA, HS was classified as moderate-severe-very severe (PGA $>3$ ) and as minimal-mild HS (PGA < 3). Besides, data on disease duration were also gathered in all the patients.
All the participants provided information on demographic features, past medical history, and current and prior systemic therapy. Traditional CV risk factors were defined as previously reported [14]. Body height and weight, body mass index (BMI), waist circumference (WC), systolic blood pressure (BP) and diastolic BP were measured in all patients and controls. BMI was calculated as weight $(\mathrm{kg}) /[\text { height }(\mathrm{m})]^{2}$. Blood samples were collected after 12-h fasting in all participants. Glucose, glycated haemoglobin $\left(\mathrm{HbA}_{1 \mathrm{c}}\right)$, triglycerides, serum total cholesterol (TC), high-density lipoprotein cholesterol (HDL-C), low-density lipoprotein cholesterol (LDL-C), high-sensitivity C-reactive protein (hs-CRP) levels and erythrocyte sedimentation rate (ESR) were measured. Metabolic syndrome was diagnosed by the presence of three or more criteria according to the National Cholesterol Education Program's Adult Treatment Panel III (ATP III) [17]. The homeostatic model assessment for insulin resistance (HOMA-IR) was calculated using the following formula: fasting insulin level $(\mu \mathrm{IU} / \mathrm{ml}) \times$ fasting glucose level (mg/dl)/405. IR was defined as an elevated HOMA-IR value of $>2.5$.

Serum levels of adipokines, omentin-1 and apelin, were measured by enzyme-linked immune-sorbent assay (ELISA) commercial kits (Sigma-Aldrich Co. LLC, St. Louis, MO, USA). Intra- and inter-assay coefficients of variation (CV) were $<10 \%$ both adipokines.

\section{Statistical analysis}

Results were expressed as numbers (percentage), mean \pm standard deviation (SD) or median and interquartile range (IQR), as appropriate. To compare quantitative and qualitative variables Mann-Whitney $U$-test and $\chi^{2}$ or Fisher test, respectively, were used. To evaluate the relationship between serum adipokines and HOMA-IR, Pearson's correlation was used. Moreover, forward stepwise multivariable logistic regression models were built to assess the potential association between both serum adipokines and IR, HS risk and HS severity. Bonferroni correction for multiple assessments was performed.

\section{Results}

Table 1 shows the main demographic, clinical and laboratory data of HS patients and controls. Both study groups did not differ in terms of age, gender, triglyceride levels, $\mathrm{HbA}_{1 \mathrm{c}}$, LDL-c and prevalence of dyslipidaemia and hypertension. Patients with HS had significantly higher $\mathrm{BMI}$, waist perimeter, systolic blood pressure (SBP) and diastolic blood pressure (DBP), serum glucose and insulin levels, HOMA-IR values, and prevalence of IR and MS than controls. Moreover, HS patients had lower serum HDL-c levels than the control group. Serum omentin-1 levels were significantly higher in subjects with HS compared to controls (513.1 (364.9-631.1) vs 309.0 (238.1-377.4); $p<0.0001)$. However, serum apelin levels 
Marcos A. González-López, J. Gonzalo Ocejo-Vińals, Cristina Mata, Diego Díaz, Sandra Guiral, Virginia Portilla, Alfonso Corrales, M. Carmen González-Vela, Miguel A. González-Gay, Ricardo Blanco, José L. Hernández

Table 1. Demographic, clinical and laboratory findings of patients with HS and controls

\begin{tabular}{|c|c|c|c|}
\hline Parameter & HS patients $(n=78)$ & Controls $(n=61)$ & $P$-value \\
\hline Age [years] & $42.6 \pm 11.7$ & $45.4 \pm 12.9$ & 0.18 \\
\hline Sex, male (\%) & 47.4 & 51.6 & 0.62 \\
\hline Active smoking (\%) & 65.4 & 19.4 & $<0.0001$ \\
\hline $\mathrm{BMI}\left[\mathrm{kg} / \mathrm{m}^{2}\right]$ & $29.4 \pm 5.4$ & $26.5 \pm 4.5$ & 0.001 \\
\hline Waist perimeter $[\mathrm{cm}]$ & $99.6 \pm 13.9$ & $91.4 \pm 13.6$ & 0.001 \\
\hline $\mathrm{SBP}[\mathrm{mm} \mathrm{Hg}]$ & $132.6 \pm 16.4$ & $124.2 \pm 15.8$ & 0.003 \\
\hline $\mathrm{DBP}[\mathrm{mm} \mathrm{Hg}]$ & $82.0 \pm 13.8$ & $76.9 \pm 8.3$ & 0.01 \\
\hline hs-CRP [mg/dl] & $0.42(0.17-0.89)$ & $0.10(0.10-0.20)$ & $<0.0001$ \\
\hline $\mathrm{HbA}_{1 \mathrm{c}}(\%)$ & $5.2 \pm 0.6$ & $5.2 \pm 0.3$ & 0.61 \\
\hline LDL-c [mg/dl] & $116.6 \pm 32.4$ & $122.4 \pm 29.2$ & 0.28 \\
\hline $\mathrm{HDL}-\mathrm{c}[\mathrm{mg} / \mathrm{dl}]$ & $46.0(41.0-56.3)$ & $52.5(46.8-69.5)$ & 0.001 \\
\hline Triglycerides [mg/dl] & $99.4 \pm 48.1$ & $96.5 \pm 66.3$ & 0.76 \\
\hline Fasting plasma glucose [mg/dl] & $94.9 \pm 13.7$ & $89.1 \pm 8.1$ & 0.004 \\
\hline Fasting plasma insulin $[\mu \mathrm{IU} / \mathrm{ml}]$ & $10.6(5.5-16.9)$ & $7.4(4.9-10.7)$ & 0.006 \\
\hline HOMA-IR & $2.2(1.0-3.7)$ & $1.5(0.9-2.3)$ & 0.005 \\
\hline Insulin resistance (\%) & 46.2 & 19.4 & 0.001 \\
\hline Hypertension (\%) & 17.9 & 14.5 & 0.59 \\
\hline Dyslipidaemia (\%) & 13.0 & 16.1 & 0.60 \\
\hline Metabolic syndrome (\%) & 34.5 & 11.5 & 0.002 \\
\hline Omentin [ng/ml] & $513.1(364.9-631.1)$ & $309.0(238.1-377.4)$ & $<0.0001$ \\
\hline Apelin [ng/ml] & $569.6(292.7-829.7)$ & $465.1(286.1-762.9)$ & 0.28 \\
\hline
\end{tabular}

BMI - body mass index, $H b A_{I C}$ - glycated haemoglobin, SBP-systolic blood pressure, DBP-diastolic blood pressure, hs-CRP-high-sensitive C-reactive protein, $L D L-c-l o w$-density lipoprotein, HDL-c - high-density lipoprotein, HOMA-IR - homeostatic model assessment for insulin resistance. Values are expressed as mean \pm SD or median (interquartile range) as appropriate. Final significant $p$-value after Bonferroni correction for multiple assessments is $p=0.0023$. Thus, significant values are marked in bold.

Table 2. Adjusted risk factors for HS development

\begin{tabular}{lccc}
\hline Parameter & $\beta$-coefficient & OR $(95 \% \mathrm{Cl})$ & $P$-value \\
\hline Active smoking, yes & 2.444 & $11.52(3.97-33.37)$ & $<0.0001$ \\
\hline BMI $\left[\mathrm{kg} / \mathrm{m}^{2}\right]$ & 0.155 & $1.17(1.05-1.29)$ & 0.004 \\
\hline Insulin resistance, yes & 2.029 & $7.61(1.99-29.08)$ & 0.003 \\
\hline Omentin $[\mathrm{ng} / \mathrm{ml}]$ & 0.012 & $1.012(1.007-1.017)$ & $<0.0001$ \\
\hline
\end{tabular}

Adjusted by age, sex, BMI, insulin resistance, active smoking and serum omentin levels.

were not significantly different between both groups. After Bonferroni correction for multiple assessments was applied, significant differences persisted for active smoking habit, BMI, waist perimeter, serum hs-CRP, and $\mathrm{HDL}-\mathrm{c}$ levels, insulin resistance, metabolic syndrome, and omentin serum levels. The differences in omentin-1 serum concentrations between HS patients and controls remained statistically significant after adjusting for age, sex, and BMI (data not shown).

According to the HS-PGA, patients with a more severe HS (PGA $\geq 3$ ) were slightly older and had a longer duration of HS, higher serum levels of hs-CRP, triglycerides, $\mathrm{HbA}_{1 \mathrm{c}}$ and more prevalence of IR and MS compared to those with a PGA score $<3$. We did not find any association between serum omentin-1 or apelin concentrations and disease severity (data not shown).

Omentin-1 did not show any significant correlation with age, sex, BMI, serum HDL-c or LDL-c cholesterol, triglycerides, and glucose or insulin levels. Serum omentin-1 concentrations were correlated positively with serum hsCRP levels $(r=0.205 ; p=0.015)$, but this association was cancelled after adjusting by age, sex and BMI. Moreover, no correlation between omentin-1 levels and HOMA-IR, IR and MS was observed. Furthermore, we did not find any significant correlation between circulating apelin concentrations and any clinical and laboratory parameters. 
The results of multivariable logistic regression analysis, adjusted for age, sex, BMI, smoking status and IR are shown in Table 2. Thus, increased serum concentrations of omentin- 1 were related to an increased risk of HS (OR $=1.012,95 \% \mathrm{Cl}: 1.007-1.017 ; p=0.0001)$. Adding some other covariables such as hypertension, dyslipidaemia, physical exercise, and MS to the model, did not change these results.

\section{Discussion}

Our study revealed that serum omentin-1 levels were significantly higher in HS patients compared to controls, whilst no differences were found regarding serum apelin levels. Furthermore, omentin-1 was related to the risk of HS. To our knowledge, this is the first study that assesses these two recently identified adipokines in HS patients.

Omentin-1 (intelectin-1) is an adipokine highly and selectively expressed in visceral adipose tissue [15]. It acts centrally to modulate IR, body weight and inflammation. It has been considered that omentin-1 exerts insulinsensitizing effects by enhanced insulin-mediated glucose transport in human adipocytes [15]. Thus, several studies have demonstrated that serum omentin-1 concentrations inversely correlate with IR and obesity $[18,19]$. Moreover, in patients with T2DM, serum omentin-1 levels were found to be significantly lower than controls, which may suggest that insulin and glucose might down-regulate omentin-1 concentrations [19]. Nevertheless, we did not find any significant association between serum omentin-1 levels and fasting glucose, HOMA-IR and BMI either in HS patients or in controls. In this sense, the increased serum omentin-1 concentrations found in our HS patients with a high prevalence of IR and obesity could be considered as an unexpected finding. However, it should be noted that increased levels of omentin-1 have also been reported in patients with non-alcoholic fatty liver disease (NAFLD) [20], another condition closely related to IR and MS. Interestingly, we have recently reported a high prevalence of NAFLD in HS patients [21].

In our study, elevated serum omentin-1 levels were associated with an increased risk of HS. Omentin-1 has usually been identified as an adipokine with anti-inflammatory effects. However, recent experimental investigations have demonstrated that this adipokine also exerts pro-inflammatory actions [22]. Moreover, omentin-1 serum levels have been found to be increased in other chronic inflammatory conditions such as psoriatic arthritis [23] and juvenile chronic arthritis [24]. We do not have a clear explanation for the increased serum omentin-1 levels in our HS patients. However, a plausible mechanism may be the up-regulation of omentin-1 expression mediated by pro-inflammatory cytokines that are upregulated in $\mathrm{HS}$ and involved in the pathogenesis of this disease. In this regard, Niersmann et al. in a proteomic analysis of mature adipocytes, showed that the most po- tential upstream regulators pointed towards pro-inflammatory effects of omentin-1, were pro-inflammatory cytokines such as IL-1 $\beta$ or TNF- $\alpha$ [22]. Alternatively, another possible explanation may be that omentin-1 could play a role in the pathogenesis of HS. Currently, it is considered that the multiprotein complex inflammasome is an important activator of inflammation in HS [10]. Interestingly, it has been demonstrated that omentin- 1 is able to activate inflammasome [22] and, inflammasome activation promotes IL-1 $\beta$ cytokine secretion, which is highly activated and plays a pivotal role in HS pathogenesis [10, 25]. In this respect, it should also be noted that a positive correlation between high levels of omentin-1 and high levels of IL-1 $\beta$ has been recently documented [26].

On the other hand, apelin is another new adipokine secreted by different cellular types, including adipocytes and endothelial cells [16]. The synthesis of apelin in adipocytes is strongly up-regulated by insulin [27]. Furthermore, apelin is closely associated with IR and elevated serum apelin levels have been found in obese and hyperinsulinemic mice and humans [27]. Moreover, it is known that apelin exerts an anti-inflammatory action by several mechanisms, including the reduction of proinflammatory cytokines [28]. In this regard, several studies have shown significantly decreased apelin levels in patients with chronic inflammatory conditions such as RA [29] or psoriasis [30]. In our study, however, apelin serum concentrations did not differ between HS patients and controls, suggesting a lack of pathogenic effect of this adipokine on this disorder.

Our study has the inherent limitations of its crosssectional design. Furthermore, another limitation could be that the International Hidradenitis Suppurativa Severity Score System (IHS4) to assess HS severity was not used. Nevertheless, we think that this study provides new data about the possible role of serum omentin-1 dysregulation in HS pathogenesis.

\section{Conclusions}

We found that serum omentin-1 but not apelin levels were significantly increased in HS patients. Furthermore, high serum omentin-1 levels were associated with HS risk. Further research is needed to clarify the potential role of these new adipokines in HS.

\section{Acknowledgments}

José L. Hernández and Ricardo Blanco shared senior authorship.

This study was funded through an unrestricted grant provided by AbbVie.

\section{Conflict of interest}

The authors declare no conflict of interest. 


\section{References}

1. Zouboulis CC, Desai N, Emtestam L, et al. European S1 guideline for the treatment of hidradenitis suppurativa/acne inversa. J Eur Acad Dermatol Venereol 2015; 29: 619-44.

2. Fimmel S, Zouboulis CC. Comorbidities of hidradenitis suppurativa (acne inversa). Dermatoendocrinol 2010; 2: 9-16.

3. Shlyankevich J, Chen AJ, Kim GE, Kimball AB. Hidradenitis suppurativa is a systemic disease with substantial comorbidity burden: a chart-verified case-control analysis. J Am Acad Dermatol 2010; 71: 1144-50.

4. Vilanova I, Hernández JL, Mata C, et al. Insulin resistance in hidradenitis suppurativa: a case-control study. J Eur Acad Dermatol Venereol 2018; 32: 820-4.

5. Phan K, Charlton O, Smith SD. Hidradenitis suppurativa and metabolic syndrome - systematic review and adjusted meta-analysis. Int J Dermatol 2019; 58: 1112-7.

6. Phan K, Charlton O, Smith SD. Hidradenitis suppurativa and diabetes mellitus: updated systematic review and adjusted meta-analysis. Clin Exp Dermatol 2019; 44: e126-32.

7. González-López MA, Hernández JL, Lacalle M, et al. Increased prevalence of subclinical atherosclerosis in patients with hidradenitis suppurativa. J Am Acad Dermatol 2016; 75: 329-35.

8. Negus D, Ahn C, Huang W. An update on the pathogenesis of hidradenitis suppurativa: implications for therapy. Expert Rev Clin Immunol 2018; 14: 275-83.

9. Vossen ARJV, van der Zee HH, Prens EP. Hidradenitis suppurativa: a systematic review integrating inflammatory pathways into a cohesive pathogenic model. Front Immunol 2018; 9: 2965.

10. Vekic DA, Frew J, Cains GD. Hidradenitis suppurativa, a review of pathogenesis, associations, and management. Part 1. Australas I Dermatol 2018; 59: 267-77.

11. Żelechowska P, Kozłowska E, Pastwińska J, et al. adipocytokine involvement in innate immune mechanisms. J Interferon Cytokine Res 2018; 38: 527-38.

12. Malara A, Hughes R, Jennings L, et al. Adipokines are dysregulated in patients with hidradenitis suppurativa. Br J Dermatol 2018; 178: 792-3.

13. Akdogan N, Alli N, Uysal PI, et al. Visfatin and insulin levels and cigarette smoking are independent risk factors for hidradenitis suppurativa: a case-control study. Arch Dermatol Res 2018; 310: 785-93.

14. González-López MA, Vilanova I, Ocejo-Vińals G, et al. Circulating levels of adiponectin, leptin, resistin and visfatin in non-diabetic patients with hidradenitis suppurativa. Arch Dermatol Res 2020; 312: 595-600.

15. Yang RZ, Lee MJ, Hu H, et al. Identification of omentin as a novel depot-specific adipokine in human adipose tissue: possible role in modulating insulin action. Am J Physiol Endocrinol Metab 2018; 290: E1253-61.

16. Antushevich H, Wójcik M. Review: apelin in disease. Clin Chim Acta 2018; 483: 241-8.

17. National Cholesterol Education Program (NCEP) Expert Panel on Detection, Evaluation, and Treatment of High Blood Cholesterol in Adults (Adult Treatment Panel III). Third report of the National Cholesterol Education Program (NCEP) expert panel on detection, evaluation, and treatment of high blood cholesterol in adults (Adult Treatment Panel III). Final report. Circulation 2002; 106: 3143-21.

18. de Souza Batista CM, Yang RZ, Lee MJ, et al. Omentin plasma levels and gene expression are decreased in obesity. Diabetes 2007; 56: 1655-61.
19. As Habi A, Sadeghi M, Arab A, Hajianfar H. The association between omentin and diabetes: a systematic review and meta-analysis of observational studies. Diabetes Metab Syndr Obes 2019; 12: 1277-86.

20. Yilmaz Y, Yonal O, Kurt R, et al. Serum levels of omentin, chemerin and adipsin in patients with biopsy-proven nonalcoholic fatty liver disease. Scand J Gastroenterol 2011; 46: 91-7.

21. Durán-Vian C, Arias-Loste MT, Hernández JL, et al. High prevalence of non-alcoholic fatty liver disease among hidradenitis suppurativa patients independent of classic metabolic risk factors. J Eur Acad Dermatol Venereol 2019; 33: 2131-6.

22. Niersmann C, Hauck SM, Kannenberg JM, et al. Omentinregulated proteins combine a pro-inflammatory phenotype with an anti-inflammatory counter-regulation in human adipocytes: a proteomics analysis. Diabetes Metab Res Rev 2019; 35: e3074.

23. Xue Y, Jiang L, Cheng Q, et al. Adipokines in psoriatic arthritis patients: the correlations with osteoclast precursors and bone erosions. PLoS One 2012; 7: e46740.

24. Cantarini L, Simonini G, Fioravanti A, et al. Circulating levels of the adipokines vaspin and omentin in patients with juvenile idiopathic arthritis, and relation to disease activity. Clin Exp Rheumatol 2011; 29: 1044-8.

25. Witte-Händel E, Wolk K, Tsaousi A, et al. The IL-1 pathway is hyperactive in hidradenitis suppurativa and contributes to skin infiltration and destruction. J Invest Dermatol 2011; 139: 1294-305.

26. Zabetian-Targhi F, Mirzaei K, Keshavarz SA, Hossein-Nezhad $\mathrm{A}$. Modulatory role of omentin-1 in inflammation: $\mathrm{cy}-$ tokines and dietary intake. J Am Coll Nutr 2016; 35: 670-8.

27. Boucher J, Masri B, Daviaud D, et al. Apelin, a newly identified adipokine up-regulated by insulin and obesity. Endocrinology 2005; 146: 1764-71.

28. Zhou H, Yang R, Wang W, et al. Fc-apelin fusion protein attenuates lipopolysaccharide-induced liver injury in mice. Sci Rep 2018; 8: 11428.

29. Di Franco M, Spinelli FR, Metere A, et al. Serum levels of asymmetric dimethylarginine and apelin as potential markers of vascular endothelial dysfunction in early rheumatoid arthritis. Mediators Inflamm 2012; 2012: 347268.

30. Capo A, Di Nicola M, Costantini E, et al. Circulating levels of Apelin 36 in patients with mild to moderate psoriasis. G Ital Dermatol Venereol 2020; 155: 646-51. 